\title{
Televisión digital: Cambio tecnológico o tecnología para el cambio
}

\author{
Gerardo Arias Carbajal (Universidad de Lima, Perú) \\ Recibido 4/9/2007 \\ Aprobado 12/10/2007
}

RESUMEN: El presente artículo da cuenta de los primeros pasos que se vienen dando en el Perú para transitar hacia la televisión digital. Sin dejar de lado el factor técnico que es necesario evaluar para adoptar un estándar de televisión digital terrestre (TDT), se enfatiza en la necesidad de reflexionar y debatir sobre el tipo de televisión que queremos para el futuro. Se discute el impacto que tendría en nuestro país la televisión de alta definición y el sistema multicasting, dos componentes centrales de la televisión digital.

Palabras clave: Televisión digital - políticas de televisión - industria televisiva televisión de alta definición - multicasting.

\section{Digital Television: Technological change or technology for change}

Summary: This article is about Peru's firsts steps to digital television. It emphasizes the need to think and debate about what type of television we want for the future (without leaving out the technical side witch must be evaluated to have a standard for digital terrestrial television or DTT) and this way turn this technological change into a chance to update all of the Peruvian television industry. Having said that, it discusses what kind of impact would, high definition television and the multicasting system (both key components of digital television), have in our country.

Key words: Digital television - television politics - television industry - high definition television - multicasting. 
T a introducción de la televisión di$\amalg$ gital terrestre (TDT) en el Perú puede ser parte de un rutinario y simple cambio tecnológico en el devenir de cualquier industria o una verdadera oportunidad para transformar notablemente el modo en que vemos, sentimos y pensamos la televisión.

Sin caer en la ingenuidad de la neutralidad de las tecnologías, pero tampoco en la falsa ilusión del cambio sustancial solo por la tecnología, es necesario debatir intensamente qué televisión queremos y de qué manera utilizaremos las posibilidades que acarrea consigo la televisión digital para acercarnos a ese ideal que hayamos delineado. La necesaria elección de un estándar de televisión digital terrestre, siendo importante, sería menos dramática si se tiene en claro el horizonte televisivo que hayamos trazado.

Con la constitución y puesta en marcha de la Comisión Multisectorial encargada de recomendar el estándar de televisión digital terrestre, ${ }^{1}$ el Perú ha iniciado el largo proceso de tránsito de la televisión analógica a la televisión digital de acceso libre, aunque el camino digital ya se ha iniciado en la televisión de pago vía la transmisión satelital (2005) y de cable (2007) a través de algunas de las empresas que operan dichos sistemas en el país. ${ }^{2}$

Como en la mayoría de países en los que se viene trabajando en el proceso de adopción - o se ha adoptado

Cuadro 1

Tenencia de TV en hogares Perú urbano

\begin{tabular}{lcccc}
\hline Hogares & & Total & $\begin{array}{c}\text { Lima } \\
\text { metropolitana }\end{array}$ & $\begin{array}{c}\text { Interior } \\
\text { urbano }\end{array}$ \\
\hline Con televisión & $(\%)$ & 93 & 97,7 & 89,9 \\
& Miles & $4.289,9$ & $1.825,5$ & $2.464,4$ \\
Sin televisión & $(\%)$ & 7 & 2,3 & 10,1 \\
& Miles & 321,5 & 43,9 & 277,6 \\
\hline Universo hogares & & & & \\
Perú urbano & & $4.611,4$ & $1.869,4$ & 2.742 \\
\hline
\end{tabular}

Fuente: CPI, mayo-junio 2007.

1 Constituida por Resolución Suprema 010-2007-MTC, publicada en el diario oficial El Peruano el 21 de febrero del 2007. El plan de trabajo elaborado por dicha comisión fue aprobado por el Ministerio de Transportes y Comunicaciones el 28 de julio, y contó con un plazo de 120 días para emitir su informe.

2 Se trata de las empresas DirecTV (satélite) y Cable Mágico (cable). 
ya - de un estándar de TDT, la importancia de la elección está ligada precisamente a las posibilidades de acceso libre que trae consigo la TDT, lo que permitiría que se logren niveles de cobertura similares a los que hoy se cuenta con la televisión analógica (cuadros 1 y 2), con la ventaja de recibir una mejor calidad de imagen y sonido y con menos posibilidades de interferencia en la transmisión, serio problema en la recepción televisiva de nuestro país (cuadro 3).

Cuadro 2

Tenencia de TV b/n - color y parque de TV Perú urbano*

\begin{tabular}{lccc}
\hline TV en hogares & Total & $\begin{array}{c}\text { Lima } \\
\text { metropolitana }\end{array}$ & $\begin{array}{c}\text { Interior } \\
\text { urbano }\end{array}$ \\
\hline Parque de TV (miles) & $7.272,5$ & $3.513,3$ & $3.759,2$ \\
Televisores a color (\%) & 85,4 & 97 & 88 \\
Televisores en blanco y negro (\%) & 17,2 & 9,8 & 24,8 \\
Núm. de televisores por hogar & & & \\
1 televisor & 54,7 & 43 & 63,4 \\
2 televisores & 28,3 & 32,4 & 25,3 \\
3 televisores & 12,1 & 17,1 & 8,4 \\
4 televisores & 3,4 & 5,2 & 2 \\
5 televisores & 1,6 & 2,3 & 0,9 \\
Promedio de TV por hogar & 1,7 & 1,9 & 1,5 \\
\hline
\end{tabular}

Fuente: CPI, mayo-junio 2007.

* Según Centrum al Día, la importación de televisores creció un 15,5\% el 2006, y alcanzó los 124,3 millones. Desde el año 2006 hasta julio de este año habrían ingresado cerca de 60 mil televisores plasma y LCD.

\section{Cuadro 3}

Calidad de recepción de canales de señal abierta Perú urbano (\%)

\begin{tabular}{lcccccc}
\hline \multirow{2}{*}{ Desempeño } & \multicolumn{7}{c}{ Canales de señal abierta } \\
& Frec. Latina & América TV & Panam. TV & TV Perú & ATV & Red Global \\
\hline Imagen & & & & & & \\
$\quad$ Buena & 57,7 & 66,2 & 62,7 & 65,1 & 74,6 & 38,4 \\
$\quad$ Con interferencia & 8,7 & 6,7 & 9,5 & 6,8 & 5,8 & 6,5 \\
$\quad$ Reflejada & 3,5 & 3,8 & 3,4 & 2,6 & 3,3 & 2,3 \\
$\quad$ Lluviosa & 14,8 & 10,8 & 14,9 & 14,4 & 9,7 & 9,4 \\
$\quad$ No hay señal & 15,3 & 12,5 & 9,5 & 11,1 & 6,6 & 43,4 \\
Sonido & & & & & & \\
$\quad$ Nítido & 77 & 81,4 & 79 & 80,8 & 86 & 48,8 \\
$\quad$ Con interferencia & 5,4 & 5,2 & 9,4 & 5,8 & 5,7 & 5,3 \\
$\quad$ Muy malo/No se escucha & 17,6 & 13,4 & 11,6 & 13,4 & 8,3 & 45,6 \\
\hline
\end{tabular}

Fuente: CPI, mayo-junio 2007. 
Si bien en esta etapa la atención está centrada en la elección de uno de los estándares de TDT, no hay que perder de vista la complejidad de todo el proceso, que implicará cambios en el marco regulador y una implementación que debe estar aparejada con un mecanismo de transición hasta que ocurra el "apagón analógico", todo lo cual puede tardar entre cinco y diez años. En efecto, a partir de la adopción del estándar de TDT, todas las empresas televisivas deberán iniciar el proceso de migración, en unos plazos que deben ser estipulados en su oportunidad por el Gobierno, y empezar a transmitir en analógico y digital (simulcasting).

Los usuarios tienen la alternativa de adquirir un televisor digital o continuar con sus actuales receptores, para lo cual deben adquirir decodificadores (set top box) que les permitan seguir viendo televisión aun cuando se haya producido el "apagón analógico". Por esta razón, uno de los puntos críticos de la elección del estándar de TDT es el tipo de decodificador que se va a utilizar y su costo, a efectos de acelerar el proceso de migración digital. El debate sobre la televisión que queremos y que el país requiere, sin embargo, aún no tiene el volumen, contundencia y amplitud necesarios para involucrar al conjunto de la opinión pública en este proceso de cambio.

\section{La elección del estándar de televisión digital terrestre}

La digitalización es un proceso mediante el cual sonidos e imágenes (además de otro tipo de información) se transforman en códigos binarios, comprimiéndose las señales originales, lo que permite la convergencia de contenidos y plataformas - de allí que la televisión digital pueda ser transmitida por ondas hertzianas, cables ópticos o emisiones satelitales$\mathrm{y}$ un ahorro importante en el uso del espectro radioeléctrico. De acuerdo con Gustavo Gómez: “Los distintos estándares aprovechan este ahorro de diversas maneras, en función de diferentes modelos de implementación, que a su vez están relacionados con diferentes modelos de negocios" ${ }^{3}$

Al no haber tecnología neutra y ser necesario elegir un estándar de TDT, es importante tener en claro las posibilidades que ofrecen los distintos estándares reconocidos por la Unión Internacional de Telecomunicaciones (UIT): ${ }^{4}$ Advanced Television System Commitee (ATSC, norteamericano), Digital Video Broadcasting (DBV, europeo) e Integrated Services Digital Broadcasting (ISDB, japonés). El desa-

3 GÓMEZ GERMANO, Gustavo. La radio y la televisión en la era digital, 2007.

4 El otro estándar existente ha sido desarrollado por la República Popular China. 
rrollo de cada uno de ellos, signado por las características y necesidades de sus propios contextos, ha generado que pongan acentos distintos a las potencialidades por explotar en esta tecnología.

Así, el estándar norteamericano pone énfasis en la televisión de alta definición (HDTV), y responde de esta manera a las demandas de los broadcasters de ese país, interesados tanto en dar mayor competitividad a la señal abierta, visto el mayor desarrollo de la televisión de pago, como en frenar el ingreso de cualquier nuevo operador. Aclaremos que de explotarse el HDTV, tendría que usarse todo el ancho de banda (6 a $8 \mathrm{MHz}$ de acuerdo a cada país) que se asigne para la transmisión digital, eliminándose la posibilidad de multicasting, esto es la transmisión de varios contenidos a través de ese mismo ancho de banda, opción esta última que constituye el énfasis que trae consigo el estándar europeo, aunque dejando de lado la transmisión en alta definición para dar paso a lo que se conoce como televisión digital estándar (SDTV), que sin alcanzar los niveles de resolución de HDTV supera el ofrecido por la televisión analógica tanto en calidad de imagen como en sonido. De este modo, el sistema europeo - atendiendo a las características de la mayor parte de sus países, donde la televisión de señal abierta sigue teniendo una notable importancia frente a la de pago, así como a las necesidades de desarro- llo de televisoras públicas y comunitarias - ha generado un estándar que justamente optimiza el uso del espectro radioeléctrico y posibilita el multicasting $\mathrm{y}$, de ser posible, el ingreso de nuevos operadores.

El estándar japonés tiene como característica principal la recepción portátil y móvil, debido al fuerte desarrollo de las telecomunicaciones y en especial de la telefonía celular en dicho país.

Sin embargo, más allá de las peculiaridades reseñadas para cada estándar, es importante notar que, tal como ha sido señalado por diversos analistas y los propios representantes de los diversos estándares, se viene trabajando en las mejoras de estos, de modo tal que en el futuro cercano convergirán en las mismas posibilidades, quedando relegadas las diferencias a eficiencias técnicas antes que a posibilidades disminuidas; a costos de implementación según las economías de escala que cada estándar logre adquirir; así como al soporte financiero y técnico que puedan brindar a un país en el proceso de implementación. La elección final de un estándar para un país, además de estos factores, está sometida también a criterios políticos, geopolíticos y de tratados de comercio. Allí están los casos de México, Canadá y otros países de Centroamérica, que han optado por el estándar ATSC, por su cercanía y tratados comerciales con Estados Unidos; los casos de Uruguay $\mathrm{y}$, aparentemente, Argentina, 
que han optado por el estándar DVB, en vista de los planes de cooperación que han logrado con los representantes europeos; $y$, por último, el de Brasil, que optó por el estándar japonés ISDB, en vista de la flexibilidad otorgada para que pueda adaptarlo a sus requerimientos pero, sobre todo, a la posibilidad de desarrollar su industria de hardware televisivo necesaria para la implementación del cambio.

\section{Las posibilidades de la televisión digital}

De acuerdo con la Comisión Multisectorial encargada de recomendar al Ministerio de Transportes y Comunicaciones el estándar de televisión digital terrestre que debe ser adoptado en el Perú, ${ }^{5}$ los beneficios que trae la TDT son los siguientes:

- Alta calidad de imagen y sonido, debido a la ausencia de ruido en las señales digitales.

- Una mejor recepción en las bandas atribuidas al servicio de televisión.

- Posibilita la recepción portátil y móvil de las señales transmitidas.

- Es posible transportar en el mismo ancho de banda que ocupa un canal analógico de $6 \mathrm{MHz}$, varios programas de televisión digital con definición estándar (SDTV), o transportar al menos uno de alta definición (HDTV).

- Permite la recepción de hasta seis canales de sonido digital.

- Convergencia con redes de telefonía móvil.

- Acceso a internet.

- Innumerables servicios interactivos de multimedia y teleinformática.

Todos estos beneficios son posibilidades que pueden activarse dependiendo del estándar elegido y, sobre todo, de las políticas que sobre la televisión digital se implementen. Subyace a estos beneficios la posibilidad de que la industria televisiva pueda ingresar en nuevos modelos de negocio. Abordemos dos aspectos centrales en las posibilidades de la TDT, considerando el impacto que tendrían en la televisión peruana.

\section{Televisión de alta definición}

La notable calidad de imagen que trae consigo la HDTV nos permitiría disfrutar plenamente de producciones internacionales que vienen procesándose en esta modalidad, especialmente en formatos en los que la calidad visual es muy valorada, tales como películas, documentales o eventos especiales. Pero no hay televisión en HDTV sin una mayor inversión en va-

5 Comisión Multisectorial. [en línea]. "Plan de trabajo" $<$ http://www:mtc. gob.pe/portal/tdt/plan.html . 
lores de producción, lo que implica tiempo y dinero. En ese sentido, la HDTV no parece viable para la producción de programas de un canal de señal abierto típico, habitualmente centrado en programas de no ficción, en vivo y de escasos valores de producción, y, por el contrario, podría desnudar las imperfecciones y precariedades de la puesta en escena (tales como la escenografía, vestuario y maquillaje).

En otras palabras, veríamos los mismos programas que actualmente nos ofrecen los canales de señal abierta en transmisión analógica, pero, para bien o para mal, con una mejor resolución de imagen.

Esta opción, sin embargo, es la que, en opinión de los broadcasters peruanos, ${ }^{6}$ convendría desarrollar en el país, de acuerdo con el modelo de negocio de la televisión de señal abierta. Debido a este modelo de negocio, se tendría que optar por la HDTV por dos razones:

- Hacer más competitiva la señal abierta, pues esta se financia por una inversión publicitaria que cada día se diversifica en nuevas plataformas mediáticas.

- Hacer viable el proyecto económico de los canales actuales, restrin- giendo el ingreso de nuevos operadores en vista del pequeño tamaño del mercado.

Frente a lo anterior es conveniente reflexionar un poco sobre lo que ocurre en la televisión comercial actual, modelo en el que se inscribe la televisión peruana. Partamos de un hecho fundamental: el negocio de la televisión es construir audiencias que interesen a los anunciantes, no necesariamente producir contenidos, aunque estos son un medio para tal fin. Por este motivo, muchas cadenas de televisión, en diversas partes del mundo, dejan en manos de productores independientes la creación de contenidos nuevos, salvo que ellas mismas consideren que pueden producir contenidos más eficaces. Claro está que la producción más de flujo - es la del día a día, de actualidad, como noticieros, debates y ciertos magacines - habitualmente queda a cargo de las cadenas, y la producción más de fondo - aquella que mantiene su valor luego de la primera exhibición, como novelas, series y miniseries - es encargada o comprada a terceros. En un contexto internacional, las productoras independientes pugnan por lograr interesar a las cadenas por el contenido que han diseñado, producido y muchas veces "testeado", sabiendo

6 Presentaciones de la Asociación de Radio y Televisión y la Sociedad Nacional de Radio y Televisión ante la Comisión Multisectorial de TDT [en línea]. www.mtc.gob.pe/portal/ tdt/exposiciones.html. 
que su salida por una cadena supone no solo la inversión de esta en el proyecto, sino también una presencia en el mercado que les puede permitir "engancharse" con nuevos proyectos. En ese sentido, y de acuerdo con lo anterior, el rol de las productoras independientes es concentrarse en la exploración de nuevos contenidos a través de una inversión creativa que las cadenas no podrían asumir: la diversidad de productoras que desarrollan proyectos nuevos supera largamente el aporte de cualquier departamento de producción o creación que una cadena pueda crear. De este modo, las cadenas se concentran en su core competence, esto es crear audiencias seleccionando los contenidos que conviene explotar y añadiendo operaciones de valor como son la programación, promoción y packaging televisivo.

Lo descrito líneas arriba es válido en lo central para nuestra realidad, aunque con algunos matices adicionales. Los canales nacionales esperan lograr estos contenidos de fondo a niveles de inversión mucho más bajos, debido a las siguientes razones:
- la alta capacidad de negociación que tienen los canales de televisión en vista de ser un mercado de pocos operadores; ${ }^{7}$

- la necesidad que tienen los productores independientes de tener una salida por sus pantallas;

- la existencia de una infraestructura ociosa ante la reducción de la producción publicitaria (como el caso de Chroma, que ingresó como realizadora en alianza con Michel Gómez y otros productores para aprovechar sus equipos);

- la aparición de un mayor número de jóvenes profesionales de la producción y realización, y

- las bajas tarifas publicitarias a las que se ha acostumbrado el anunciante nacional.

Si bien nuestras productoras, tal como ocurre en otras partes del mundo, invierten en la exploración creativa, no realizan eficazmente un proceso básico que es el filtrado y depuración de ideas, así como la utilización de ciertos mecanismos para "testear" sus proyectos antes de emprender

7 Descartando a TV Perú, canal estatal, y RBC, de escasa y precaria programación, se puede considerar, para estos efectos, básicamente cuatro operadores: ATV (que también opera Red Global, ambos en manos del mexicano Ángel Gonzales); América Televisión (administrado por Plural TV, que cuenta también con el canal de cable local N, empresa del Grupo El Comercio); Frecuencia Latina (administrado judicialmente por Baruch Ivcher y en litigio con los hermanos Winter); Panamericana Televisión (administrado judicialmente por Genaro Delgado Parker y en litigio con la familia Schutz). 
aventuras mayores. $Y$ esta es una gran diferencia con otros mercados. La otra diferencia es que, al estar enfocados en la reducción de costos por la presión de los canales, no están logrando obras con los suficientes valores de producción que permitan su eventual exportación y, como contraparte, acentúan mucho más el carácter local de la propuesta.

Finalmente, la otra gran diferencia con otros mercados más desarrollados es que los canales de televisión nacionales, aun cuando recurren a la producción independiente, no se concentran ni gerencian adecuadamente lo que hemos señalado como su competencia principal, esto es construir audiencias. Un solo hecho basta para evidenciar esto último: ante la presencia de la oferta de cable, los canales de señal abierta se han replegado en los públicos populares, no porque sepan cómo llegar a ellos sino porque prejuzgan que son más fáciles de complacer - lo que se traduce en menores montos de inversión - y además consideran que allí tienen menos competencia. Ambos criterios no son axiomas válidos y eso puede corroborarse con los resultados de la programación: los públicos populares no son necesariamente más fáciles de com- placer y la carencia de cable no quiere decir que no tengan otras alternativas de consumo (allí está, en primer lugar, la piratería de la señal de cable, las películas que pueden verse en DVD piratas, internet o la vida cotidiana misma). El mensaje es claro: si no hay nada que valga la pena ver, los niveles de encendido bajan.

Pero la sola mejora de la calidad técnica de la imagen y el sonido no hará más competitiva a una televisora, y el interés inicial que pueda despertar la HDTV puede apagarse pronto si no se invierte efectivamente en el desarrollo de nuevos contenidos que renueven la programación peruana. Más aún cuando el espectador tiene acceso - o lo tendrá en un futuro mediato- a nuevas formas de entretenimiento audiovisual, que serán críticas en el futuro de la televisión: DVD, DVR, ipods, celulares, internet y la propia televisión de pago (véanse cuadros 4 y 5).

Por otro lado, si bien la inversión publicitaria en el Perú es reducida, ${ }^{8}$ en gran parte se debe a la propia gestión de los canales de televisión peruanos, que no siempre han sabido desarrollar nuevas y valiosas audiencias, y a la precariedad legal en que se halla la mayoría de ellos, en disputa

8 Es necesario considerar, sin embargo, que la inversión publicitaria en televisión se ha recuperado en los últimos cinco años, pasando de 63 millones de dólares (2001) a 121 millones de dólares (2006). El cable, en el mismo periodo, ha pasado de 9 a 15 millones de dólares. Fuente: CPI. 
permanente entre los propietarios por el control judicial de las estaciones televisivas.

No hay que olvidar, además, que la televisión comercial en el Perú está enmarcada en una economía de libre mercado, por lo que, aun cuando se mantuvieran niveles bajos de inversión publicitaria, no se sostendría el argumento del "pequeño mercado de la televisión" para impedir el ingreso de nuevos operadores.

Otro factor para tomar en cuenta, de cara al futuro, es que si bien los

\section{Cuadro 4}

Tenencia de VHS/DVD Perú urbano

\begin{tabular}{|c|c|c|c|}
\hline Hogares que & Total & $\begin{array}{c}\text { Lima } \\
\text { metropolitana }\end{array}$ & $\begin{array}{l}\text { Interior } \\
\text { urbano }\end{array}$ \\
\hline No tienen VHS & 80,9 & 77,2 & 83,7 \\
\hline Sí tienen VHS & 19,1 & 22,8 & 16,3 \\
\hline $1 \mathrm{VHS}$ & 18,3 & 21,6 & 15,8 \\
\hline $2 \mathrm{VHS}$ & 0,8 & 1,2 & 0,5 \\
\hline Promedio VHS por hogar & 1 & 1,1 & 1 \\
\hline No tienen DVD & 34,8 & 25,4 & 41,7 \\
\hline Sí tienen DVD & 65,2 & 74,6 & 58,3 \\
\hline $1 \mathrm{DVD}$ & 57,2 & 62,5 & 53,2 \\
\hline 2 DVD & 6,4 & 9,5 & 4,2 \\
\hline 3 DVD a más & 1,6 & 2,6 & 1 \\
\hline Promedio DVD por hogar & 1,2 & 1,2 & 1,1 \\
\hline \multicolumn{4}{|l|}{ Base estadística: } \\
\hline Total hogares con TV & 2.824 & 586 & 2.238 \\
\hline
\end{tabular}

Fuente: CPI mayo-junio 2007.

\section{Cuadro 5}

Tenencia de TV cable en hogares Perú urbano

\begin{tabular}{llccc}
\hline Hogares & Total & $\begin{array}{c}\text { Lima } \\
\text { Metropol. }\end{array}$ & $\begin{array}{c}\text { Interior } \\
\text { urbano }\end{array}$ \\
\hline Sin TV cable & & & & \\
& $\%$ & 72,3 & 55,5 & 83,8 \\
& Miles & $3.334,4$ & $1.037,4$ & 2.297 \\
Con TV cable & & & 44,5 & 16,2 \\
& $\%$ & 27,7 & 832 & 445 \\
\hline & Miles & 1.277 & $1.869,5$ & 2.742 \\
\hline Universo hogares & $4.611,5$ & & \\
\multicolumn{2}{l}{ Perú urbano } & & &
\end{tabular}

Fuente: CPI mayo-junio 2007. 
montos de inversión publicitaria son bajos, lo que debe importar es la relación de estos con el producto bruto interno (PBI), así como la inversión publicitaria per capita. Y como puede observarse (cuadro 6), todavía falta mucho para alcanzar el promedio latinoamericano. A un mayor desarrollo de valor agregado de los medios, y en este caso de la televisión, podríamos tener una inversión publicitaria que haga viable la presencia de más operadores. Tampoco debe perderse de vista que la introducción de la TDT trae consigo la posibilidad de ingresar a nuevos modelos de negocio que permitirán incrementar los ingresos de los canales de televisión.

\section{Multicasting}

Lograr un uso más eficiente del espectro radioeléctrico transmitiendo en SDTV, cuya calidad de imagen y sonido sigue siendo superior a la actual televisión analógica, puede permitir que en el mismo ancho de banda con el que hoy operan los canales de televisión en VHF se pueda ofrecer cuatro o más contenidos. Con lo cual no solo se amplían las posibilidades de programación a las que puede acceder un consumidor, también puede modificarse la estructura misma de la industria televisiva en el Perú. El modo definitivo en que se configure dependerá de la discusión sobre el modelo de

\section{Cuadro 6}

Inversión publicitaria en América Latina 2006

\begin{tabular}{lrrrrc}
\hline País & \multicolumn{1}{c}{$\begin{array}{c}\text { Inversión } \\
\text { publicitaria (1) }\end{array}$} & $\begin{array}{c}\text { Cantidad de } \\
\text { habitantes (2) }\end{array}$ & $\begin{array}{c}\text { Inversión public. } \\
\text { per cápita (1) }\end{array}$ & PBI & $\begin{array}{c}\text { Inversión } \\
\text { publicitaria/ } \\
\text { PBI (\%) }\end{array}$ \\
\hline Argentina & $1.748 .870,37$ & 38.800 .000 & 45,07 & $187.035 .000,00$ & 0,94 \\
Brasil & $7.244 .276,14$ & 186.400 .000 & 38,86 & $784.509 .000,00$ & 0,92 \\
Chile & $890.270,50$ & 16.300 .000 & 54,62 & $107.703 .000,00$ & 0,83 \\
Colombia & $1.498 .577,23$ & 44.900 .000 & 33,38 & $108.461 .000,00$ & 1,38 \\
Ecuador & $685.497,22$ & 13.710 .234 & 50,00 & $39.600 .000,00$ & 1,73 \\
México & $3.933 .600,00$ & 107.000 .000 & 36,76 & $811.002 .000,00$ & 0,49 \\
Perú & $320.100,00$ & 28.303 .000 & 11,31 & $77.358 .000,00$ & 0,41 \\
Uruguay & $150.450,00$ & 3.400 .000 & 44,25 & $14.182 .000,00$ & 1,06 \\
Venezuela & $898.637,70$ & 26.600 .000 & 33,78 & $134.628 .000,00$ & 0,67 \\
Total Latam (3) & $17.370 .279,16$ & 465.413 .234 & 37,32 & $2.264 .478 .000,00$ & 0,77 \\
\hline
\end{tabular}

Fuentes:

(1) Estimaciones AAAP basadas en la información suministrada por las distintas cámaras, asociaciones de agencias de publicidad y empresas de medición de los países que intervienen.

(2) Fondo Monetario Internacional (FMI) / Comisión Económica para América Latina y el Caribe (Cepal).

(3) Cifras totales referidas a los nueve países participantes.

Elaboración: Asociación Argentina de Agencias de Publicidad (AAAP). 
televisión que se pretende para el país y de la legislación que se sancione al respecto. Es necesario advertir que en el caso peruano, el Ministerio de Transportes y Comunicaciones ha establecido la reserva en la banda de 470-584 MHz (canales 14 al 32), para el servicio de televisión digital terrestre. Estas son las nuevas licencias sobre las que deberá legislarse. En todo caso, conviene tomar en cuenta las alternativas manejadas en la experiencia de otros países: ${ }^{9}$

- Licitación pública de las nuevas licencias. Las nuevas bandas de televisión digitales son consideradas un servicio nuevo que no tendría que asignarse directamente a los operadores de televisión analógica (Francia, Inglaterra).

- Régimen de licencias canal por canal en vez de entregar todo el ancho de banda $(6 \mathrm{MHz})$ en bloque a los concesionarios, a efectos de que emitan varios contenidos (Suecia, Finlandia).

- Concesiones nacionales, regionales y locales por separado (España).

Algunas de estas modalidades permitirían dinamizar la industria televisiva con el ingreso de nuevos opera- dores; ofrecer nuevos enfoques televisivos, tanto por lo anterior como por la necesaria búsqueda de nuevos creadores de contenido, que contarían ahora con mayores posibilidades de desarrollo en vista de la mayor cantidad de operadores; acompañar el proceso de regionalización que vive el país implementando televisoras que respondan a este objetivo, propiciando una verdadera descentralización informativa y comunicacional; y lograr que, efectivamente, la televisión contribuya con la educación y la cultura, a través de la entrega de licencias a instituciones de la sociedad civil que puedan producir con solvencia una programación de este tipo.

Si bien podría parecer que las opciones de multicasting y HDTV son opuestas entre sí, en el actual escenario de convergencia tecnológica la flexibilidad es un atributo básico que hay que buscar en el estándar que se elija. Esta flexibilidad permite que cada operador configure, en cada momento, sus transmisiones de acuerdo con su propio plan de negocios. ${ }^{10} \mathrm{Co}-$ mo consecuencia,

... no existe realmente una dicotomía entre la modalidad de alta definición y la opción por el multicasting; entre la

9 GODOY ETCHEVERRY, S. “Desafíos regulatorios para la adopción de la televisión digital terrestre en Chile", 2005.

10 De acuerdo con la evaluación de estándares realizada por la Pontificia Universidad Católica de Chile, octubre del 2006. 
oferta de programación televisiva de máxima resolución espacial y la oferta de múltiples programas de definición estándar y/o nuevos servicios de información. ${ }^{11}$

Es necesario, entonces, que en se evalúe con detenimiento esta flexibilidad de los estándares, para atender así tanto a la propia industria televisiva actual como a las necesidades comunicativas reseñadas.

\section{Hacia la exploración televisiva ${ }^{12}$}

Visto así el panorama de la televisión peruana, el advenimiento de la televisión digital puede contribuir no solo a la optimización del uso del espectro radioeléctrico, haciéndolo menos escaso, sino también a propiciar que corra la misma suerte que otros recursos naturales, cuyas regulaciones han buscado su mejor utilización y preservación, a través de diferenciar condiciones de explotación de condiciones de exploración. ${ }^{13}$

En el campo televisivo, la Constitución y las leyes apenas esbozan mínimas condiciones de explotación, como son el respeto a los derechos hu- manos, la contribución a la educación y la cultura, la pluralidad informativa, etcétera. Aspectos todos ellos fundamentales para la formación de una ética ciudadana, entendida como una sociedad pluralista de ciudadanos autónomos que, pese a sus diferencias, comparten un conjunto de valores.

Como es evidente, estas mínimas condiciones de explotación son transgredidas cuando se denigra o estigmatiza a las minorías; cuando se relativiza el derecho a la imagen y la intimidad de las personas; y cuando la información requerida por el ciudadano es escamoteada, tergiversada o presentada desde un solo ángulo.

Claro está que pueden haber otras condiciones de explotación que se impongan a las estaciones de televisión - por ejemplo, unos indicadores sobre el nivel educativo y cultural de sus contenidos-, pero ganaríamos mucho como sociedad si tan solo se cumplieran las que permiten construir una sociedad de ciudadanos.

Si son poco exigentes y escasamente evaluadas las condiciones de explotación, las condiciones de exploración son inexistentes en el campo televisi-

11 Consejo Nacional de Televisión de Chile. Situación de la televisión digital en el mundo e implicancias en Chile. Santiago de Chile, abril del 2006.

12 El planteamiento central de esta parte ha sido desarrollado con otros fines en ARIAS, Gerardo. "De la explotación a la exploración televisiva". Gaceta Cultural 19, 2006.

13 Una condición de explotación en el campo petrolero sería la preservación del medio ambiente; mientras que una condición de exploración es la inversión en la búsqueda de nuevos pozos. 
vo. Y si algo requiere explorar la televisión peruana son nuevas formas de hacer televisión; nuevos enfoques y puntos de vista; innovaciones narrativas; irrupciones en nuevos campos temáticos; estrategias narrativas que hagan entretenido lo cultural y lo educativo y que tornen educativos y culturales los contenidos de entretenimiento; nuevos tratamientos periodísticos que amplíen el horizonte informativo de los ciudadanos antes que reducírselo a simples lugares comunes.

El solo hecho de discutir sobre la televisión que queremos, del modelo de asignación de frecuencias que debería seguirse camino a la televisión digital puede generar la puesta en marcha de nuevas condiciones de explotación. La apuesta por el multicasting propiciaría que se atienda una condición fundamental para que puedan hacerse efectivas las condiciones de exploración señaladas, esto es propiciar que en la programación tengan cabida diversos productores de contenido, en su más amplia pluralidad y procedencia, que compitan entre sí para desarrollar espacios de televisión en la búsqueda de mejorar la oferta televisiva.

De este modo, los canales de televisión podrían concentrarse en generar valor agregado a esos contenidos en aras de construir unas audiencias que interesen a los anunciantes, pero sobre la base de atender, en primera instancia, al ciudadano que está detrás de cada uno de los puntos de sintonía.

\section{Bibliografía}

ARIAS CARBAJAL, Gerardo. “De la explotación a la exploración televisiva". Gaceta Cultural 19. Lima: Instituto Nacional de Cultura, mayo del 2006.

Asociación Argentina de Agencias de Publicidad. "Informe de la inversión publicitaria 2006" [en línea]. $<$ http://www.aaap.org>.

Centrum-PUCP. "Mercado de electrodomésticos". Centrum al Día. Boletín electrónico de negocios. Lima, 15 de febrero del 2007.

Comisión Multisectorial de TDT en el Perú. "Plan de trabajo" [en línea]. $<$ http://www.mtc.gob.pe/portal/t $\mathrm{dt} /$ inicio.html>. Julio del 2007.

Consejo Consultivo de Radio y Televisión (Perú). Especial sobre la televisión digital terrestre [en línea]. $<$ http://www.concortv.gob.pe>.

Consejo Nacional de Televisión de Chile. Situación de la televisión digital en el mundo e implicancias para Chile. Santiago de Chile, abril del 2006.

CPI SAC. "Inversión publicitaria 2001-2006" [en línea]. <http:// www.cpi.com.pe>. 
GODOY ETCHEVERRY, Sergio. “Desafíos regulatorios para la adopción de la televisión digital terrestre en Chile". Documento elaborado para el Consejo Nacional de Televisión de Chile. Santiago de Chile, noviembre del 2005.

GÓMEZ GERMANO, Gustavo. La radio y la televisión en la era digital. Centro de Competencia en Co- municación para América Latina. Bogotá: Friedrich Ebert Stiftung, febrero del 2007.

Pontificia Universidad Católica de Chile. "Análisis de los estándares de transmisión de televisión digital terrestre y su aplicabilidad al medio nacional". Escuela de Ingeniería, octubre del 2006. 